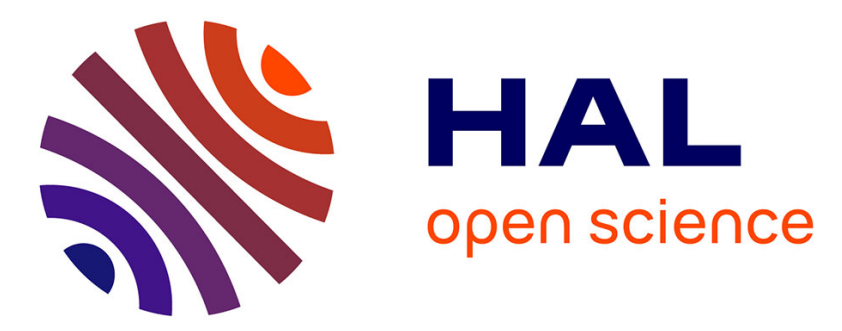

\title{
Une querelle orientaliste : la réception controversée du Zend Avesta d'Anquetil-Duperron en France et en Angleterre \\ Claire Gallien
}

\section{- To cite this version: \\ Claire Gallien. Une querelle orientaliste : la réception controversée du Zend Avesta d'Anquetil- Duperron en France et en Angleterre. Littératures classiques, 2013, 81 (2), pp.257-268. 10.3917/li- cla.081.0257 . halshs-01611577}

\section{HAL Id: halshs-01611577 \\ https://shs.hal.science/halshs-01611577}

Submitted on 6 Oct 2017

HAL is a multi-disciplinary open access archive for the deposit and dissemination of scientific research documents, whether they are published or not. The documents may come from teaching and research institutions in France or abroad, or from public or private research centers.
L'archive ouverte pluridisciplinaire HAL, est destinée au dépôt et à la diffusion de documents scientifiques de niveau recherche, publiés ou non, émanant des établissements d'enseignement et de recherche français ou étrangers, des laboratoires publics ou privés. 


\section{UNE QUERELLE ORIENTALISTE : LA RÉCEPTION CONTROVERSÉE DU ZEND AVESTA D'ANQUETIL-DUPERRON EN FRANCE ET EN ANGLETERRE \\ Claire Gallien}

Armand Colin | « Littératures classiques »

$2013 / 2 N^{\circ} 81 \mid$ pages 257 à 268

ISSN 0992-5279

ISBN 9782200928599

Article disponible en ligne à l'adresse :

http://www.cairn.info/revue-litteratures-classiques1-2013-2-page-257.htm

\section{Pour citer cet article :}

Claire Gallien, « Une querelle orientaliste : la réception controversée du Zend Avesta d'Anquetil-Duperron en France et en Angleterre », Littératures classiques 2013/2 $\left(\mathrm{N}^{\circ} 81\right)$, p. 257-268.

DOI 10.3917/licla.081.0257

Distribution électronique Cairn.info pour Armand Colin.

(C) Armand Colin. Tous droits réservés pour tous pays.

La reproduction ou représentation de cet article, notamment par photocopie, n'est autorisée que dans les limites des conditions générales d'utilisation du site ou, le cas échéant, des conditions générales de la licence souscrite par votre établissement. Toute autre reproduction ou représentation, en tout ou partie, sous quelque forme et de quelque manière que ce soit, est interdite sauf accord préalable et écrit de l'éditeur, en dehors des cas prévus par la législation en vigueur en France. Il est précisé que son stockage dans une base de données est également interdit. 


\title{
Claire Gallien
}

\author{
UNE QUERELLE ORIENTALISTE : \\ LA RÉCEPTION CONTROVERSÉE \\ DU ZEND AVESTA D'ANQUETIL- \\ DUPERRON EN FRANCE ET EN \\ ANGLETERRE
}

La distinction entre la controverse, la querelle et le débat peut paraître ténue. En effet, ils appartiennent tous trois à la catégorie de l'échange verbal et opposent deux ou plusieurs personnes de manière souvent vive. Cependant, si le débat présuppose un même niveau des intervenants, la controverse implique que l'un d'entre eux ou plusieurs personnes à la fois remettent en cause les qualités morales ou intellectuelles de ceux qui en sont l'objet. Ainsi, la controverse induit un questionnement quant à la fiabilité et à la validité du travail ou des propos tenus par la ou les personne[s] ciblée(s), et provoque une perte de confiance et de légitimité ${ }^{1}$. Le Zend Avesta, ensemble de textes sacrés zoroastriens, traduits pour la première fois en Europe par l'indianiste français Abraham Hyacinthe AnquetilDuperron et publiés en 1771, a suscité une controverse dont la victime est le traducteur, et l'on compte parmi ses détracteurs des philosophes, des théologiens et des orientalistes.

Avant même la parution de l'ouvrage, des érudits français expriment leurs réserves quant à l'authenticité ou l'intérêt des textes rapportés par Anquetil de Surate, où l'indianiste avait passé plusieurs années à collecter des informations et des copies de manuscrits auprès de la communauté parsie. Diderot, par exemple,

1 Sur la relation entre élaboration du savoir et rapport de confiance entre savants, voir notamment S. Shapin, A Social History of Truth. Civility and Science in Seventeenth-Century England, Chicago, Chicago University Press, 1994. 


\section{Claire Gallien}

loue la qualité du travail d'Anquetil, notamment dans son article « Zend-Avesta » de l'Encyclopédie (t. XVII, 1751-1765), mais il considère, dans l'article « Perses », que les récits de la vie de Zoroastre sont fictifs et refuse de croire que ce dernier ait pu être l'auteur d'un tel galimatias. De même, l'abbé Ladvocat écrit en 1760 dans son Dictionnaire historique portatif que les manuscrits zoroastriens sont déjà à la Bibliothèque du Roi mais qu'ils ne sont d'aucune utilité. Il précise : « Feu M. Otter en avait commencé la traduction mais il y trouva tant de fables qu'il abandonna son projet ${ }^{2}$. 》 L'épisode est repris par Anquetil dans son « Discours préliminaire 》 au Zend Avesta, où il explique avoir vérifié les textes utilisés par Otter et en avoir conclu qu'il ne s'agissait pas des mêmes car ceux-ci n'étaient pas écrits en avestique.

En réalité, beaucoup craignaient qu'Anquetil n'ait été dupé par la fourberie de prêtres parsis. En effet, l'époque était riche en production de faux manuscrits orientaux, qui régalaient les amateurs de contes pseudo-orientaux et déroutaient les savants. Les recherches de Ludo Rocher et d'Urs App, par exemple, ont permis de démontrer que l'Ézour Vedam, dont Voltaire s'inspire dès 1760, n'était pas un traité védique mais un faux réalisé par Jean Calmette, missionnaire jésuite en Inde du Sud ${ }^{3}$. Étant donné que peu d'érudits étaient en mesure de vérifier l'authenticité de certaines productions, notamment en sanscrit ou en avestique, la tentation était grande de produire des faux. Dès lors, chaque nouvelle découverte comme celle d'Anquetil pouvait rapidement porter à controverse.

La controverse ainsi engagée en France à propos du Zend Avesta prend une dimension européenne avec la parution de la Lettre à $A^{* * *} d u P^{* * *}$ publiée en 1771 par l'orientaliste anglais Sir William Jones. Si ce dernier ne réapparaît plus ensuite dans l'échange, d'autres prennent sa place et continuent d'alimenter le débat concernant la validité du travail de l'indianiste français. Ainsi, en France, certains philosophes, comme Voltaire, Diderot et Grimm, lui reprochent de mettre à mal leur lecture anticléricale de la religion des Guèbres. D'autres parmi ses amis et mécènes - tels Condorcet et Turgot, le sinologue De Guignes, mais également son traducteur allemand Kleuker, le numismate Tychsen et le philosophe Herder - le soutiennent. La communauté orientaliste est également divisée : Jones, Richardson, Chardin, Volney, ou encore Christoph Meiners en Allemagne, le dénoncent publiquement.

2 J.-B. Ladvocat, Dictionnaire historique portatif, Paris, Vve Didot, 1760, t. II, p. 998.

3 U. App, The Birth of Orientalism, Philadelphie, University of Pennsylvania Press, 2010, p. 372-407 ; L. Rocher, Ezourvedam : A French Veda of the Eighteenth Century, Amsterdam, J. Benjamins, 1984, t. I. Rocher identifie Calmette comme l'un des auteurs possibles de l'Ézour, aux côtés de Roberto de Nobili, Antoine Mosac, ou Pierre Martin. 
Ces derniers l'accusent d'avoir plagié le travail du professeur d'études orientales à Oxford, Thomas Hyde, de s'être moqué de l'érudition du professeur d'hébreu à Oxford, Thomas Hunt, et d'avoir menti sur l'authenticité des manuscrits utilisés pour sa traduction. La controverse ne prend fin qu'en 1826, lorsque l'orientaliste hollandais Ramsus Rask prouve, dans son essai Sur l'ancienneté et l'authenticité $d u$ Zend Avesta ${ }^{4}$, l'originalité des manuscrits utilisés par Anquetil ; authenticité confirmée par Burnouf en 1833.

La chronologie de la controverse du Zend Avesta a déjà été établie ${ }^{5}$, et je ne reviendrai que brièvement sur celle-ci. L'objet de cet article est de montrer que, contrairement à ce qui a été écrit à son sujet, cette controverse a été vecteur de création et a permis le développement des connaissances. En effet, les biographes de Jones, qu'il s'agisse de Garland Cannon ou de Michael J. Franklin, semblent gênés par la nature de sa lettre, qui détone par rapport à ses méthodes habituellement sérieuses d'érudit. Ils préfèrent la considérer comme une pièce à part, relevant davantage de la satire que de la recherche orientaliste ${ }^{6}$. De même, ils semblent unanimes pour condamner les effets pervers qu'elle entraîna, retardant d'un demi-siècle la recherche sur le zoroastrisme et sur les langues de la Perse antique? . L'hypothèse de travail que je voudrais retenir ici est que la controverse a eu des conséquences plus complexes sur les poursuites de la recherche qu'un simple effet de retardement. En une première partie, j'identifie les points précis de ce volet anglais de la controverse, tels qu'ils sont débattus de la fameuse lettre de Jones. En seconde partie, j'analyse les conséquences de la controverse pour les études orientalistes, non seulement concernant la poursuite du travail d'Anquetil et de ses détracteurs, comme Jones ou Richardson, mais également concernant le développement de la discipline elle-même.

\section{Chronologie et enjeux de la controverse du Zend Avesta}

Le parcours orientaliste d'Anquetil-Duperron débute à Paris, à la Bibliothèque royale, où il découvre le calque d'un manuscrit avestique dont l'original est

4 Über das Alter und die Echtheit der Zend-Sprache und des Zend-Avesta, Berlin, Duncker \& Humblot, 1826.

5 Voir notamment R. Schwab, Vie d'Anquetil-Duperron, Paris, E. Leroux, 1934, p. 85-104; L. Valensi, «Anquetil-Duperron », dans Fr. Pouillon [dir.], Dictionnaire des orientalistes de langue française, Paris, Karthala, 2008, p. 21-23.

6 G. Cannon, The Life and Mind of Oriental Jones, Cambridge, Cambridge University Press, 1990, p. $42-43$; M. J. Franklin, Orientalist Jones, Oxford, Oxford University Press, 2011, p. 74.

? G. Cannon, op. cit., p. 42. 


\section{Claire Gallien}

conservé à la Bodleian Library. Cette première découverte le mène sur le chemin de l'Inde du sud, où il séjourne entre août 1755 et mars 1761. En Inde, il apprend le persan, acquiert des connaissances en malabar, bengali, pehlevi et avestique grâce aux lettrés locaux, et se procure des copies de textes sacrés zoroastriens, dictés par les destours (prêtres) de la communauté parsie de Surate. Anquetil rentre en Europe sur un navire britannique et s'arrête à Londres et à Oxford, où il rencontre notamment l'orientaliste Thomas Hyde, avec qui il s'entretient très probablement de ses récentes découvertes. Une fois de retour en France, il dépose ses 180 manuscrits à la Bibliothèque royale et prépare son récit de voyage, qu'il publie en 1762 .

Dans un premier temps, la réception du travail d'Anquetil est positive. En Angleterre, The Annual Register for the Year 1762 publie une recension des plus élogieuses ${ }^{8}$. En France, Anquetil gagne la reconnaissance du monde savant en étant élu membre associé de l'Académie royale des inscriptions et belles-lettres en 1765 , ce qui lui permet de donner lecture de ses découvertes devant les autres membres de l'Académie et de publier dans les Mémoires de l'Académie et dans le Journal des sçavans.

La controverse du Zend Avesta ne débute que plus tard, au moment de la parution des premiers articles académiques d'Anquetil en rapport avec sa traduction des textes sacrés. En effet, si Anquetil publie séparément la Relation abrégée du voyage en 1762, soit un an après son retour de Surate, ce n'est qu'à partir de 1768 qu'il contribue aux Mémoires de l'Académie des inscriptions et belles-lettres, ainsi qu'au Journal des sçavans au sujet du zoroastrisme, en proposant des extraits du Zend Avesta ${ }^{9}$ et des articles en rapport avec la religion et les langues de la Perse antique. Ces articles anticipent sur certains points de la controverse. Dans ces deux essais « sur les anciennes langues de la Perse », il démontre que le zend est la langue mère du pazend, du pahlavi et du farsi, et corrige les imprécisions de

\footnotetext{
$\mathbf{8}$ « II est impossible de ne pas être impressionné par le nombre de découvertes contenues dans cet ouvrage [...]. Personne ne peut être plus respecté que ce véritable virtuoso, qui affronta tous les dangers et les difficultés afin de promouvoir un savoir utile et de donner encore davantage de matière aux spéculations des érudits. 》 [ «It is impossible not to be struck with the importance of many of the discoveries contained in it [...]. No character can be more respectable, than that of a true virtuoso, who braves every danger and difficulty in order to promote useful knowledge, and to increase materials of speculation in the learned world. »] [E. Burke [éd.), Annual Register for the Year 1762, Londres, 1763, p. 101 ; nous traduisons).

9 Le Journal des sçavans diffusa d'abord un « Prospectus du Zend Avesta » (août 1769, p. 566-568), puis le « Premier extrait» du Zend Avesta (nov. 1771, p. 709-716), le « Second extrait 》 (janv. 1772, p. 41-48), et le « Troisième extrait » (mars 1772, p. 142-149).
} 
Thomas Hyde dans son Histoire religieuse des anciens Perses (Historia religionis veterum persarum, 1700 ${ }^{10}$. De même, dans l'essai sur le «Système théologique des Mages ${ }^{11}$ », Anquetil compare ce qu'a écrit Plutarque avec les « anciens livres que les Parses attribuent à Zoroastre » et prouve la justesse des remarques de l'historien romain, tout en les augmentant au moyen des sources originales. Anquetil $y$ attaque à nouveau Hyde et considère les erreurs transmises par celui-ci.

Ces articles sont en quelque sorte des premières versions ou premiers extraits avant la traduction complète du Zend Avesta en 1771. L'ouvrage est en effet beaucoup plus fourni et comprend deux tomes répartis sur trois volumes. Le premier volume (t. I, partie I) reprend le récit du voyage d'Anquetil à Surate, expliquant comment il rencontre la communauté parsie et obtient de trois destours (Dastur Darab bin Suhrab, Dastur Kavus bin Faraydun et Manuchihrji Seth) qu'ils lui dictent les textes sacrés du zoroastrisme. Le deuxième volume (t. I, partie II] contient la notice commentée des manuscrits «Zends, Pehlvis, Persans \& Indiens 》 déposés par Anquetil-Duperron à la Bibliothèque royale, le sommaire des deux tomes, le récit de la vie de Zoroastre, et le contenu du Vendidad Sadé, constitué de trois recueils de prières : l'Izechné, le Vispered, et le Vendidad. La traduction des textes est toujours accompagnée d'une préface, apportant des renseignements sur leur contenu, leur fonction et sur l'histoire de leur transmission en Asie. Le troisième volume (t. II) comprend la traduction commentée des leschts Sadés, contenant différents types de prières, du Si-Rouzé, et du Boun-Dehesch. II ajoute à ces traductions deux lexiques : l'un zend, pahlavi et français, et l'autre pahlavi, persan et français. Enfin, l'ouvrage se termine par une « exposition des usages civils et religieux des Parses » et du « système cérémonial et moral des Livres Zends et Pehlvis ».

Révélant des sources jusqu'alors inconnues, mais avançant sur un terrain déjà en partie défriché par des voyageurs érudits comme Tavernier et Chardin, et par des orientalistes comme Hunt et Hyde en Angleterre, le Zend Avesta d'Anquetil est rapidement dénoncé pour son manque d'authenticité, d'originalité et d'intérêt. La Lettre à $A^{* * *} d u P^{* * *}$ (Londres, 1771) est l'exposition la plus complète de ces tensions. L'auteur use d'un ton systématiquement méprisant, employant sarcasmes et remarques ironiques pour condamner le travail d'Anquetil. II se moque à multiples reprises du teint « de lys et de rose » que le savant se plaint d'avoir vu flétrir pendant son voyage en Inde. II lui refuse le titre d'orientaliste, mais

10 Histoire de l'Académie royale des inscriptions et belles-lettres, avec les Mémoires de littérature tirés des registres de cette académie [1710-1843], Paris, Imprimerie Royale, 1768, t. XXXI, p. 340-392 et 393-442.

$11 \mathrm{lbid} ., 1770$, t. XXXIV, p. 376-415. 


\section{Claire Gallien}

lui donne celui d'«Archimage » des Guèbres et affirme, de manière désobligeante, que personne ne prendra la peine de le lire. L'animosité de ces propos est telle que certains critiques estiment qu'elle finit par desservir Jones et nous fait douter de sa crédibilité ${ }^{12}$. Franklin en vient même à postuler que Jones aurait écrit cette lettre pour prouver à l'élite française que lui aussi savait écrire dans le style de Voltaire. Cependant, à l'époque, calomnie et critique peuvent aller de pair, et l'association des deux n'est pas perçue comme contreproductive, comme le rappelle, en 1796, l'éditeur de la publication orientaliste Dissertations and Miscellaneous Pieces: « La vanité et la pétulance d'ANQUETIL DU PERRON [...] le poussa à écrire une lettre en français, qui est admirée pour la précision de ses critiques, la justesse de sa satire, et l'élégance de sa composition ${ }^{13}$. 》

L'animosité de Jones à l'égard d'Anquetil s'exprime également à travers des attaques personnelles : il lui reproche son ambition, sa recherche de la célébrité, son narcissisme, sa vanité, et son manque d'humanité. II met en doute la probité de l'individu en l'accusant du vol de manuscrits. II condamne également son manque de reconnaissance pour ses prédécesseurs, Hyde, Hunt et d'Herbelot. II apparaît donc qu'Anquetil dérange Jones moins pour des raisons d'incompatibilité de caractère que pour la manière dont son comportement déroge aux principes de confiance et de collaboration dans les sciences.

De plus, Jones reprend Anquetil sur la qualité de son travail. Dès les premières pages de la lettre, il lui reproche un mélange des styles abusif. Sa traduction, à force de mêler le haut et le bas, comme lorsqu'il parle de dartres et d'apozèmes, devient grotesque. Alors qu'au début du XVIII e siècle, un orientaliste aussi renommé qu'Antoine Galland pouvait revendiquer le principe de l'association du divertissement et de l'instruction comme fil conducteur de ses traductions, cette interaction semble poser problème à la fin du siècle. À ses remarques d'ordre stylistique, Jones ajoute des commentaires méthodologiques. Selon lui, Anquetil est incapable de vérifier ses sources et ses informateurs - il qualifie ses destours de charlatans. Il reproche aussi à sa traduction de fourmiller de détails qui encombrent le lecteur et révèlent un cruel manque d'esprit synthétique. À l'opposé du travail d'analyse et de la passion du détail propre aux scolastiques ${ }^{14}$, Jones

12 M. J. Franklin, op. cit.

13 « The vanity and petulance of ANQUETIL DU PERRON [...] extorted from him a letter in the French language, which has been admired for accurate criticism, just satire, and elegant composition. 》 (《An Eulogium on the Life and Writings of the Late Sir William Jones », dans Dissertations and Miscellaneous Pieces, Londres, 1796, t. III, p. v; notre traduction].

14 Jones nomme Anquetil, tour à tour, voyageur, antiquaire, avocat et scolastique, mais lui refuse toujours le titre d'orientaliste. 
indique de nouvelles règles d'écriture fondées sur les principes de clarté et de synthèse.

La seconde moitié de la lettre concerne la validité scientifique du travail d'Anquetil. Jones soutient que les textes écrits dans les langues de la Perse antique ont été détruits lors des conquêtes d'Alexandre et du calife Omar. Dès lors, les prétentions d'Anquetil ne peuvent être historiquement justifiées. Jones s'engage à corriger les erreurs d'Anquetil, notamment en prouvant que la présence de termes arabes dans le Zend Avesta contredit les dires de ce dernier sur l'antiquité des textes exhumés. II critique le manque de fiabilité de la méthode employée par le Français, qui prouve le lien entre langues zend, pahlavi et persane au seul moyen de dérivations lexicales. Enfin, Jones le corrige sur des points de sémantique, de géographie, et de chronologie - en expliquant, par exemple, qu'Hafez n'est pas le contemporain de Sadi.

La lettre de William Jones expose les divers points de la controverse du Zend Avesta. Cependant, l'orientaliste précise ne pas souhaiter s'engager dans un débat plus approfondi. En effet, il garde l'anonymat et prévient Anquetil qu'il ne donnera pas suite à l'échange. Malgré cet avertissement, une réponse à Jones est publiée dans le Journal des sçavans l'année suivante. L'auteur y relève la mauvaise foi de William Jones et questionne la validité scientifique d'un projet dont le but se limite à « tourner en ridicule » Anquetil (« Cette lettre attendue depuis long tems est moins une critique, qu'une satire ») et il dénonce « une mauvaise foi dans les citations ${ }^{15} \gg$. Même si le ton de la réponse est acerbe, l'auteur n'entend pas seulement répondre à la « satire » par l'insolence. II reprend également Jones sur des questions de fond, précisant que la présence de mots arabes dans les écrits zoroastriens n'est pas une preuve scientifique suffisante pour les classer comme postérieurs à Mahomet. À la fin de la réponse, l'auteur ajoute une remarque instructive sur la fonction de la critique, jugée « inutile \& contraire aux recherches sçavantes » si elle ne dépasse pas le niveau de l'attaque personnelle et si elle n'est pas fondée sur des arguments scientifiques. En d'autres termes, les remarques concernant la personne et ses qualités morales n'ont pas de place dans un débat érudit. Dans ce contexte particulier, le rôle de la controverse est ambigu : parvient-elle à lancer une dynamique suffisamment forte et dégagée des intérêts personnels pour permettre l'avancée des connaissances ou, au contraire, se réduit-elle à un conflit d'égos entre savants?

15 «Lettre à M. du P., dans laquelle est compris l'examen de sa traduction des livres attribués à Zoroastre », Journal des sçavans, mai 1772, p. 272. 


\section{Claire Gallien}

On peut ici avancer l'hypothèse que si la controverse ne joue pas systématiquement un rôle moteur dans le développement des connaissances, elle n'est jamais sans conséquences, tant pour la production savante de ceux qui y sont engagés que pour la structuration du champ disciplinaire affecté. Je propose donc d'étudier les résonances de la controverse du Zend Avesta dans la recherche des principaux orientalistes qui y ont pris part, à savoir Anquetil, Jones et Richardson, et dans le champ des études orientalistes lui-même.

\section{Controverse et construction du savoir}

Après sa traduction du Zend, Anquetil poursuit sa recherche d'indianiste et publie un essai de philosophie politique sur la nature des gouvernements asiatiques et la traduction d'un ensemble d'upanishads, d'après une version persane. II poursuit également son travail sur les religions et langues de la Perse antique et le diffuse dans ses discours à l'Académie des inscriptions et belleslettres, repris (souvent avec quelques années d'intervalle) dans les Mémoires de cette même académie. Même s'il ne mentionne pas explicitement ses détracteurs, le contenu de ses discours indique la volonté du savant de répondre aux attaques en renforçant ses arguments.

Par exemple, son discours de mai 1767 sur la théologie mazdéenne, publié sous le titre «Exposition du système théologique des Perses ${ }^{16}$ », peut être lu comme une réponse à ceux qui, par méconnaissance, ont dénigré ou dénaturé le mazdéisme. L'étude se veut méthodique et savante, différente donc de la lecture anticléricale qu'en avaient donnée les philosophes, comme Montesquieu dans les Lettres persanes, et opposée à la lecture moqueuse de Voltaire dans Le Philosophe ignorant (1766). De même, sa « Recherche sur le temps auquel a vécu Zoroastre ${ }^{17} 》$ apporte la preuve, contre les allégations de Diderot dans l'Encyclopédie, que Zoroastre a bel et bien existé et qu'il est l'auteur des textes sacrés en langue avestique. Le « Mémoire dans lequel on établit que les livres zends déposés à la Bibliothèque du Roi, le 15 mars 1762, sont les propres ouvrages du Zoroastre ${ }^{18} \gg$

16 Histoire de l'Académie royale des inscriptions et belles-lettres, t. XXXVII, 1774, p. 571-709.

17 Ibid., p. $710-758$.

18 Le mémoire est d'abord publié dans le Journal des sçavans de mai 1769 (p. 270-300) et de juin 1769 (p. 336-372) puis réimprimé dans les Mémoires (t. XXXVIII, 1777, p. 167-268). 
s'adresse à ceux qui, comme Jones, Diderot ou l'abbé Ladvocat, doutaient de l'originalité des manuscrits rapportés par Anqueti ${ }^{19}$ ou de leur intérêt.

De plus, la controverse a des conséquences pour la recherche philologique de Sir William Jones et de John Richardson. Anquetil avait défendu l'antiquité de son manuscrit en précisant que la présence de mots arabes dans le texte ne compromettait en rien l'ancienneté de ce dernier. Pour lui, la présence arabe dans la région bien avant l'établissement de l'islam explique ces nombreux emprunts. De plus, il explique, par le moyen de dérivations lexicales, que le farsi et le pehlevi sont des langues sœurs issues du zend (avestique). En 177?, John Richardson revient sur ce point dans Dissertations sur la langue, la littérature, et les mœurs des nations orientales (Dissertation on the Language, Literature and Manners of the Eastern Nations] et utilise ses compétences en persan et en arabe pour critiquer les thèses d'Anquetil ${ }^{20}$. II rappelle d'abord qu'aucun mot en arabe n'a été introduit en persan avant le VII siècle et donc que leur présence dans le Zend Avesta indique qu'il s'agit d'un texte plus récent. À cette considération lexicale s'ajoute une remarque d'ordre physiologique selon laquelle certains sons en avestique sont imprononçables par un persanophone. Le persan ne peut donc être dérivé du zend. Son dernier argument est d'ordre morphologique et grammatical. Richardson expose en effet que les langues zend et persane n'ont, de ce point de vue, pas la moindre ressemblance entre elles : elles ne sauraient donc être placées dans la même famille linguistique. En procédant ainsi, non seulement il critique Anquetil, mais, en outre, il met en place une méthode plus complète, et partant plus fiable, pour l'étude des langues, associant preuves morphologiques, grammaticales et physiologiques.

En 1789, Jones revient sur le Zend Avesta d'Anquetil et montre en quoi le travail du Français l'a servi dans ses propres recherches. Le système de dérivation morphologique qu'il élabore dans cet essai le conduit d'une part à distinguer ce qu'Anquetil avait confondu, à savoir le zend et le farsi (langues indo-européennes) du pahlavi (langue sémitique), et d'autre part à proposer le sanscrit comme origine du zend, et ce, grâce au glossaire d'Anquetil :

19 Bouchier en 1718 et Fraser en 1742 avaient déjà rapporté en Europe des manuscrits zoroastriens : voir J.-L. Kieffer, Anquetil-Duperron. L'Inde en France au XVIII' siècle, Paris, Les Belles Lettres, 1983, p. 12.

20 La Dissertation est publiée de manière séparée en 1777 mais elle était initialement prévue en préface du Dictionary Persian, Arabic and English (17?7-1780). Les arguments de Richardson figurent p. 11-14. 


\section{Claire Gallien}

après avoir examiné avec soin son glossaire zend, j'ai été extrêmement surpris du fait que six à sept mots sur dix sont du pur sanscrit, et que même certaines de leurs inflexions sont formées à partir des règles du Vyâcaran - comme yushmâcan, qui est le génitif pluriel de yushmad [...]. Ainsi, il s'agit d'une liste authentique de mots en zend, préservés dans des livres et par la tradition, et on peut en conclure que la langue zend était au moins un dialecte du sanscrit. ${ }^{21}$

Ainsi, les découvertes controversées d'Anquetil provoquent la recherche d'autres orientalistes qui tentent d'en rectifier les erreurs, au risque d'en produire eux-mêmes de nouvelles ${ }^{22}$. La controverse témoigne également des modifications profondes affectant la structure et les pratiques de la discipline orientaliste et marquant une phase de transition dans son histoire. En effet, l'orientalisme n'est plus conçu de la même manière dans le dernier quart du XVIII siècle qu'au début de celui-ci. La discipline se pratique largement en dehors des universités, s'appuie sur des enquêtes de terrain, et ses acteurs renoncent au savoir universel pour lui préférer une forme de connaissance synthétique, spécialisée en sous-disciplines complémentaires, et vulgarisée. Dès lors, les manières de procéder d'Anquetil, et notamment ses velléités encyclopédiques, paraissent obsolètes aux yeux de certains de ses pairs. Les trois volumes du Zend Avesta sont jugés illisibles, non seulement à cause de la longueur des textes, mais également de l'aspect hétéroclite de l'ensemble. Récit de voyage, traductions d'hymnes sacrés, prières, lexiques et notes proto-ethnologiques sont mis bout à bout sans souci de cohérence ni de clarté. Les traductions sont également augmentées à outrance par des éléments paratextuels (introductions critiques, notes de traduction, repères dans les marges et translittérations) et par des explications concernant les gestes à accomplir pendant la récitation des prières et des hymnes.

Selon Jones, cette tendance au disparate rend la traduction du Zend-Avesta peu fiable et dépassée. Trop foisonnante, mélangeant les genres entre eux, cette forme de savoir est contraire aux attentes de Jones qui appelle, quant à lui, à une forme plus spécialisée et plus précise :

On doit aimer le vrai savoir: mais toutes choses ne valent pas la peine d'être sçuës. Il est inconcevable que dans un siècle, où tous les arts se perfectionnent,

21 « [...] when I perused the Zend glossary, I was inexpressibly surprized to find that six or seven words in ten were pure Sanscrit, and even some of their inflections formed by the rules of the Vyâcaran ; as yushmâcan, the genetive plural of yushmad [...] and it follows, that the language of the Zend was at least a dialect of the Sanscrit. » (W. Jones, « On the Persians », dans Asiatic Researches, Londres, 1799, t. II, p. 53-54; nous traduisons).

22 Dans son essai de 1826, Rask expose l'erreur de Jones en prouvant que l'avestique n'est pas une forme corrompue de sanscrit. 
et toutes les sciences s'épurent, ce qu'on appelle la littérature en général, soit, par faute de choix, presque barbare. ${ }^{23}$

Jones envisage clairement la simplification (ou l'épuration, pour reprendre ses termes] comme la condition désormais sine qua non du progrès des connaissances, et appelle les études littéraires à suivre le modèle et les méthodes des sciences de la nature. Ainsi, tout savoir qui n'aurait pas d'application pratique est jugé inutile :

Ne savez-vous pas que les langues n'ont aucune valeur intrinsèque ? Et qu'un érudit pourrait savoir par cœur tous les dictionnaires qui ont jamais été compilés, et pourrait bien n'être à la fin du compte que le plus ignorant des mortels ? ${ }^{24}$

Enfin, Jones reproche explicitement à Anquetil de nombreuses approximations dans ses recherches étymologiques. Sans proposer encore de méthode plus fiable, il note néanmoins que la simple proximité lexicographique ne permet pas d'établir de manière sûre des liens de parenté entre les langues. Jones, qui n'avait exposé qu'en 1786 sa nouvelle méthode linguistique empruntée aux sciences expérimentales ${ }^{25}$, explique alors que la preuve de l'affinité entre langues s'élabore en deux temps : il faut d'abord prouver des similarités systématiques entre morphèmes, puis consolider ces remarques par des correspondances dans le comportement grammatical des langues, aussi bien au niveau de la syntaxe qu'à celui des flexions, des dérivations et des combinaisons. À la même époque, Jones, partant de l'affinité de certaines langues entre elles, développe une nouvelle approche génétique, fondée sur l'hypothèse d'une langue primitive commune à toutes les langues indo-européennes. Les insuffisances concernant la méthode employée par Anquetil confirment ainsi Jones dans la nécessité d'un changement, qui servira de fondement à la linguistique moderne.

La controverse du Zend Avesta ne s'est terminée en fait qu'en 1826. Cependant, si l'on s'intéresse à l'histoire de la réception de l'ouvrage, on remarque que la controverse aura continué à dicter les modalités de celle-ci. En effet, dans un premier temps, c'està-dire de la publication de l'ouvrage en 1771 à son attestation par Rask en 1826, son authenticité, son originalité et son intérêt sont contestés. Vient ensuite le temps de la réhabilitation, de Rask et Burnouf jusqu'à Raymond Schwab en 1950, qui désigne Anquetil comme l'initiateur en France de la « Renaissance orientale ». Aujourd'hui, si

$23 \mathrm{~W}$. Jones, Lettre à $A^{* * *} d u P^{* * *}$, Londres, 1771, p. ?.

24 Ibid., p. 11.

25 W. Jones, « Third Anniversary Discourse. Delivered 2 February 1786 », Works, Londres, 1799, vol. I, p. 19-34. 


\section{Claire Gallien}

la question de l'authenticité des manuscrits semble dépassée, on s'interroge toujours sur l'originalité du travail d'Anquetil, en analysant les conditions de production du texte, et notamment en soulignant le rôle fondamental des prêtres parsis dans son élaboration ${ }^{26}$, ou en s'interrogeant sur la portée de la recherche d'Anquetil. Jenny Rose rappelle que le travail du Français consistait essentiellement à confirmer les intuitions de ses prédécesseurs, tout en fournissant des preuves de première main ${ }^{27}$. Jean Kellens note les approximations de sa recherche, essentiellement dues à la qualité de ses sources et informateurs ${ }^{28}$. D'autres sont plus circonspects quant à la rupture épistémologique qu'incarnerait Anquetil. Wilhem Halbfass préfère le placer « entre les Lumières, le Romantisme, et l'indologie scientifique », et soutient qu'il appartient à la «préhistoire de l'indologie ${ }^{29} 》$.

Ainsi, le cas du Zend Avesta montre que la controverse a une portée qui va au-delà du conflit d'intérêt personnel ou de la dissension académique. En effet, son rôle dans la réception et dans l'élaboration du savoir est central et complexe : loin d'avoir un effet seulement inhibant, comme les biographes de Sir William Jones le soutiennent, la controverse autour du Zend Avesta a stimulé la recherche, et signale un moment de transition dans l'histoire de l'orientalisme, entre une approche ancienne (de type empiriste, à vocation encyclopédique et universelle) et une approche moderne, expérimentale, fondée sur le principe d'objectivité dans la constitution du savoir. Un tel cas montre enfin de façon flagrante que les disputes savantes - au moins - se jouent à l'échelon international.

Claire Gallien

Université Paul Valéry - Montpellier 3

IRCL - UMR 5186

26 Voir sur la question du rapport d'Anquetil aux destours, et plus généralement des orientalistes aux lettrés orientaux, M. Tavakoli-Targhi, « Orientalism's Genesis Amnesia », dans Refashioning Iran. Orientalism, Occidentalism and Historiography, New York, Palgrave, 2001, p. 18-34.

27 J. Rose, The Image of Zoroaster. The Persian Mage Through European Eyes, New York, Bibliotheca Persica Press, 2000, p. 103.

28 En effet, depuis la prise de Kirman par les Afghans en 1719, la communauté parsie de Surate n'a plus communiqué avec les zoroastriens en Iran. Privée d'échanges, elle a peiné à maintenir un niveau d'érudition suffisant sur place. De plus, Anquetil n'avait pas accès aux textes originaux mais à des copies de textes dictés et utilisait souvent le persan comme langue relais, ce qui conduit à de nombreuses approximations et remet profondément en cause la notion même de texte original. Voir J. Kellens, La Quatrième naissance de Zarathustra, Paris, Seuil, 2006, p. 19-20 ; id., «L'Avesta, Zoroastre et les sources des religions indo-iraniennes », mars 2004, consultable en ligne sur le site http://www.clio.fr/BIBLIOTHEQUE/.

29 W. Halbfass, India and Europe, Albany (NY), State University of New York Press, 1988, p. 64. 